\title{
Oscillations of Fourier Coefficients of Modular Forms
}

\section{Ram Murty}

Department of Mathematics. McGill University. 805 Sherbrooke Street, W., Montreal P.Q. Canada H3A 2 K 6

\section{Introduction}

Let $f$ be a normalized Hecke eigenform of weight $k$ for the full modular group and let $a(n)$ denote its $n^{\text {th }}$ Fourier coefficient. Let us write, for each prime $p$,

$$
a(p)=2 p^{\left(\frac{k-1}{2}\right)} \cos \theta(p) .
$$

Since we know the truth of the Ramanujan-Petersson conjecture, it follows that the $\theta(p)$ 's are real.

Inspired by the Sato-Tate conjecture for elliptic curves, Serre [14] conjectured that the $\theta(p)$ 's are uniformly distributed in the interval $[0, \pi]$ with respect to the measure $\frac{1}{\pi} \sin ^{2} \theta d \theta$. Following Serre, we shall refer to this as the Sato-Tate conjecture, there being no room for confusion.

It has been known for a long time that the truth of this conjecture implies much about the oscillatory behaviour of the Fourier coefficients. In particular, the following is implied by the Sato-Tate conjecture.

Theorem 1. For any normalized Hecke eigenform,

$$
a(n)=\Omega_{ \pm}\left(n^{\left(\frac{k-1}{2}\right)} \exp \left(\frac{c \log n}{\log \log n}\right)\right)
$$

for some $c>0$.

Theorem 1 has a long history. Hardy [2] proved that

$$
a(n)=\Omega\left(n^{\frac{k-1}{2}}\right)
$$

and Rankin [11] showed

$$
\limsup _{n \rightarrow \infty} \frac{|a(n)|}{n^{\left(\frac{k-1}{2}\right)}}=+\infty .
$$


Then Joris [5] showed that for $\delta_{k}=6 / k^{2}$, we have

$$
a(n)=\Omega\left(\frac{k-1}{n^{2}} \exp \left(c(\log n)^{\delta_{k-\varepsilon}}\right)\right) .
$$

This was improved in [1] to $\delta_{k}=1 / k$. In the special case of $k=12$, the authors in [1] showed

$$
\tau(n)=\Omega\left(n^{\frac{11}{2}} \exp \left(c(\log n)^{\frac{2}{3}-\varepsilon}\right)\right)
$$

for the Ramanujan $\tau$-function. This was improved upon slightly in [8]. For an arbitrary cusp form, which is not necessarily an eigenfunction, we have:

Theorem 2. Let

$$
f(z)=\sum_{n=1}^{\infty} a(n) e^{2 \pi i n z}
$$

be a cusp form of weight $k$ for the full modular group. Then

$$
a(n)=\Omega\left(n^{\frac{k-1}{2}} \exp \left(\frac{c \log n}{\log \log n}\right)\right)
$$

for some $c_{1}>0$.

In view of Deligne's result, Theorems 1 and 2 reflect the best possible result, apart from the values of $c$ and $c_{1}$.

Theorem 2 has an interesting application to Kloosterman sums. Define

$$
S(n, m, c)=\sum_{\substack{a(c) \\ a \bar{a}=1(c)}} e^{\frac{2 \pi i}{c}(n a+m \bar{a})} .
$$

Selberg [13] and Linnik [6] have independently conjectured that the sum, for $x \geqq(m, n)^{\frac{1}{2}+\varepsilon}$,

$$
G(x)=\sum_{c \leqq x} \frac{S(n, m, c)}{c}
$$

is $O\left(x^{\varepsilon}\right)$. We deduce from Theorem 2:

Corollary. $G(x)=\Omega\left(\exp \left(\frac{c \log x}{\log \log x}\right)\right)$.

The main ingredient in the proof of Theorems 1 and 2 is a recent result of Shahidi [15] which we now describe.

Define for each integer $m \geqq 1$,

$$
L_{m}(s)=\prod_{p} \prod_{j=0}^{m}\left(1-\frac{e^{i \theta(p)(m-2 j)}}{p^{s}}\right)^{-1} .
$$

Clearly, $L_{m}(s)$ converges for $\operatorname{Re} s>1$. It is conjectured that each $L_{m}(s)$ has an analytic continuation to the entire complex plane. Moreover, the Sato-Tate conjecture is true if and only if $L_{m}(1+i t) \neq 0$ for all real $t$ and all $m \geqq 1$ (see Serre [14]). 
The fact that $L_{1}(s)$ and $L_{2}(s)$ have an analytic continuation to the entire complex plane is classical and due to Hecke and Rankin, respectively. Shahidi [15, Theorems 4.1.1 and 4.1.2] has shown that $L_{3}(s), L_{4}(s)$, and $L_{5}(s)$ have an analytic continuation to the entire complex plane and satisfy certain functional equations.

We will need the non-vanishing of $L_{3}(s)$ and $L_{4}(s)$ on the line $\operatorname{Re} s=1$. Ogg [10] proved that if for each $r \leqq 2 m, L_{r}(s)$ has an analytic continuation to $\operatorname{Re} s>\frac{1}{2}-\delta$ for some $\delta>0$, then $L_{m}(1+i t) \neq 0$. Murty [9] showed that it suffices to have analytic continuation up to $\operatorname{Re} s \geqq 1$ for the non-vanishing result to hold. As we do not have the analytic continuation of $L_{r}(s)$ for $r \geqq 6$, we cannot deduce the non-vanishing theorem from the results cited above. We therefore prove:

Theorem 3. If $L_{r}(s)$ has an analytic continuation up to $\operatorname{Re} s \geqq \frac{1}{2}$, for $1 \leqq r \leqq 2 m$, then $L_{2 m-1}(1+i t) \neq 0$. If $L_{2 r}(s)$ has an analytic continuation up to $\operatorname{Re} s=1$, for $1 \leqq r \leqq m$, then $L_{2 m}(1+i t) \neq 0$.

Corollary. $L_{3}(1+i t) \neq 0, L_{4}(1+i t) \neq 0$, for all $t \in \mathbb{R}$.

Remark. This result is partially contained in Shahidi [15, Theorem 5.3]. Our proof is different. For our application, the fact that $L_{4}(1) \neq 0$ is crucial and this is not contained in [15].

At this stage, it seems relevant to ask the following: if it is known that $L_{r}(s)$ has an analytic continuation up to $\operatorname{Re} s=1$ for $r \leqq R$, then what can be deduced about the oscillatory behaviour of the $a(n)$ 's. An answer is supplied by the following.

Theorem 4. Suppose that $L_{r}(s)$ has an analytic continuation up to $\operatorname{Re} s \geqq \frac{1}{2}$ for all $r \leqq 2 m+2$. Then, each of the statements

(i) for any $\delta>0,-\delta<2 \cos \theta(p)<\frac{2}{\delta(m+2)}$,

(ii) for any $\varepsilon>0$,

$$
|2 \cos \theta(p)|>\sqrt{\frac{4 m+2}{m+2}}-\varepsilon,
$$

(iii) for any $\varepsilon>0,2 \cos \theta(p)>\beta_{m}-\varepsilon$, where

$$
\beta_{m}=\left\{\frac{1}{4(m+2)}\left(\begin{array}{c}
2 m+2 \\
m+1
\end{array}\right)\right\}^{\frac{1}{2 m+1}}
$$

(with a corresponding result valid for negative values of a(p)), holds for some set of primes of positive density.

Setting $\delta=\sqrt{\frac{2}{m+2}}$ in (i), we deduce:

Corollary 1. There is a positive density of primes p satisfying

$$
-\sqrt{\frac{2}{m+2}}<2 \cos \theta(p)<\sqrt{\frac{2}{m+2}} .
$$


If in theorem (ii), we set $m=1$, we have:

Corollary 2. For a positive density of primes $p$, we have

$$
|a(p)|>(\sqrt{2}-\varepsilon) p^{\frac{k-1}{2}} .
$$

It is then an easy matter to deduce the $\Omega$-theorem from this fact. The $\Omega_{ \pm}$ theorem for the Fourier coefficients of an eigenform requires a further analysis of the sign changes of the $a(p)$ 's.

Theorem 5. Let

$$
f(z)=\sum_{n=1}^{\infty} a(n) e^{2 \pi i n z}
$$

be an arbitrary cusp form belonging to any congruence subgroup. Then either $\operatorname{Re} a(p)$ or $\operatorname{Im} a(p)$ changes sign infinitely often. Moreover, there exists a small positive number $\theta$ such that the number of sign changes for $p \leqq x$ is at least ax for some $a>0$.

If all the zeroes of $L_{1}(s)$ lying in the critical strip have real part $=\frac{1}{2}$, then the number of sign changes can be improved to $\gg x^{\frac{1}{2}-\varepsilon}$.

Finer theorems concerning the sign changes of the $a(p)$ 's demand an examination of the real zeroes of $L_{1}(s)$. The following conjecture can be proved in many special cases.

Conjecture 1. Let

$$
f(z)=\sum_{n=1}^{\infty} a(n) e^{2 \pi i n z}
$$

be arbitrary cuspform with real coefficients and of weight $k$ for the full modular group. Then,

(i) $\sum_{p \leqq x} a(p) p^{-(k-1) / 2}=\Omega_{ \pm}\left(x^{1 / 2} \log \log \log x / \log x\right)$,

ii) $a(n)=\Omega_{ \pm}\left(n^{(k-1) / 2} \exp (c \log n / \log \log n)\right)$, for some $c>0$.

The first part of the conjecture would follow if $L_{1}(s)$ has no real zeroes in $\frac{1}{2}<s \leqq 1$. The second part would follow if we knew that the $\theta(p)$ 's are independently distributed for the various eigenforms. These possibilities are explored in the latter sections of the paper.

\section{Non-Vanishing of $L_{r}(s)$}

We first prove Theorem 3. We need the following lemma.

Lemma 1 (Murty [9]). Let $f(s)$ be a function satisfying the following hypotheses:

(a) $f(s)$ is holomorphic in $\sigma>1$ and non-zero there,

(b) on the line $\sigma=1, f$ is holomorphic except for a pole of order $e \geqq 0$ at $s=1$,

(c) for $\sigma>1, \log f(s)$ can be written as a Dirichlet series

with $b(n) \geqq 0$.

$$
\sum_{n=1}^{\infty} b(n) n^{-s}
$$


Then, any zero of $f(s)$ on $\sigma=1$ has order $\leqq \frac{e}{2}$.

Proof of Theorem 3. We first show $L_{2 m}(1+i t) \neq 0$. Consider

$$
f(s)=L_{0} L_{2} \ldots L_{2 m} \text {. }
$$

Then, in view of the identities

$$
\frac{1}{2}+\cos \theta+\cos 2 \theta+\ldots+\cos n \theta=\frac{\sin \left(n+\frac{1}{2}\right) \theta}{2 \sin \frac{\theta}{2}}
$$

and

$$
\cos \theta+\cos 3 \theta+\ldots+\cos (2 n-1) \theta=\frac{\sin n \theta}{2 \sin \theta}
$$

we see that

$$
\log L_{r}(s)=\sum_{n, p}\left(\frac{\sin (r+1) n \theta(p)}{\sin n \theta(p)}\right) \frac{1}{n p^{n s}}
$$

Therefore, as

$$
1+\frac{\sin 3 \theta}{\sin \theta}+\frac{\sin 5 \theta}{\sin \theta}+\ldots+\frac{\sin (2 n-1) \theta}{\sin \theta}=\left(\frac{\sin n \theta}{\sin \theta}\right)^{2}
$$

we find that $\log f(s)$ is a Dirichlet series with non-negative coefficients. Moreover, the Euler product for $L_{r}(s)$ shows that $f(s)$ does not vanish in $\sigma>1$. An application of Lemma 1 with $e \leqq 1$ gives the result. Now consider

$$
g(s)=\left(L_{0} L_{1} \ldots L_{2 m-1}\right)^{2} L_{2 m} .
$$

An easy computation reveals,

$$
\log g(s)=\sum_{n, p}\left((2 m+1)+\sum_{j=0}^{2 m-1} 2(j+1) \cos (2 m-j) n \theta(p)\right) \frac{1}{n p^{n s}} .
$$

Since,

$$
(2 m+1)+\sum_{j=0}^{2 m-1} 2(j+1) \cos (2 m-j) \theta=\left(\frac{\sin \left(m+\frac{1}{2}\right) \theta}{\sin \frac{\theta}{2}}\right)^{2},
$$

$\log g(s)$ is a Dirichlet series with non-negative coefficients. If $L_{2 m-1}(1+i t)=0, t \neq 0$, then $g(s)$ has a zero of order $\geqq 2$. As $g(s)$ has a pole of order 2 at $s=1$, we get a contradiction by Lemma 1 . If $L_{2 m-1}(1)=0$, then $g(s)$ is regular. By the well-known theorem of Landau, $\log g(s)$ has a singularity at its abscissa of convergence. As $L_{0}(s)=\zeta(s)$ has zeroes in Res $\geqq \frac{1}{2}, g(s)$ has zeroes in this half plane. Therefore, the abscissa of convergence of $\log g(s)$ is $\sigma_{0} \geqq \frac{1}{2}$, and as $g(s)$ is analytic in $\operatorname{Re} s \geqq \frac{1}{2}, \sigma_{0}$ is a zero of $g(s)$. But then $g(\sigma) \geqq 1$ for $\sigma>\sigma_{0}$. We get a contradiction by letting $\sigma \rightarrow \sigma_{0}^{+}$. This completes the proof of the theorem. 


\section{Various Bounds for Fourier Coefficients}

The non-vanishing of $L_{r}(s)$ on $\sigma=1$, allows us to deduce the following lemma which we need for the proof of Theorem 4.

Lemma 2. Suppose $L_{r}(s)$ has an analytic continuation up to $\operatorname{Re} s \geqq \frac{1}{2}$ for all $r \leqq 2 m+2$. Then

(i) for $r \leqq m+1$,

$$
\sum_{p \leqq x}(2 \cos \theta(p))^{2 r}=\frac{1}{r+1}\left(\begin{array}{c}
2 r \\
r
\end{array}\right)(1+o(1)) \frac{x}{\log x}
$$

as $x \rightarrow \infty$ and

(ii) for $r \leqq m$,

$$
\sum_{p \leqq x}(2 \cos \theta(p))^{2 r+1}=o(x / \log x)
$$

as $x \rightarrow \infty$.

Proof. From Theorem 3, we know that $L_{r}(s)$ does not vanish on the line $\sigma=1$. Therefore, by the Wiener-Ikehara Tauberian theorem, we deduce for $1 \leqq r \leqq 2 m+2$,

$$
\sum_{p \leqq x} \frac{\sin (r+1) \theta(p)}{\sin \theta(p)}=o(x / \log x)
$$

as $x \rightarrow \infty$, on using the identity

$$
\frac{\sin (r+1) \theta}{\sin \theta}=\sum_{j=0}^{r} e^{i(r-2 j) \theta} .
$$

Writing $U_{n}(\cos \theta)=\frac{\sin (n+1) \theta}{\sin \theta}$ and $T_{n}(\cos \theta)=\cos n \theta$, for $n \geqq 1$, we find for $2 \leqq r$ $\leqq 2 m+2$,

$$
\sum_{p \leqq x} T_{r}(\cos \theta(p))=o(x / \log x)
$$

because of the identity

$$
2 T_{n}(x)=U_{n}(x)-U_{n-2}(x) .
$$

Also,

$$
\sum_{p \leqq x} T_{2}(\cos \theta(p))=\left(-\frac{1}{2}+o(1)\right) \frac{x}{\log x}
$$

and

$$
\sum_{p \leq x} T_{1}(\cos \theta(p))=o(x / \log x),
$$

as $L_{1}(s)$ is regular and non-vanishing for $\operatorname{Re} s \geqq 1$.

If we define $T_{0}(x)=\frac{1}{2}$, the inverse relation for Chebychev polynomials gives (see Riordan [12, p. 54]),

$$
(2 \cos \theta)^{r}=2 \sum_{k=0}^{r^{\prime}}\left(\begin{array}{l}
r \\
k
\end{array}\right) T_{r-2 k}(\cos \theta),
$$


where $r^{\prime}=[r / 2]$. Therefore,

$$
\sum_{p \leqq x}(2 \cos \theta(p))^{r}=2 \sum_{k=0}^{r^{\prime}}\left(\begin{array}{l}
r \\
k
\end{array}\right) \sum_{p \leqq x} T_{r-2 k}(\cos \theta(p)) .
$$

By the above remarks, the inner sum is $o(x / \log x)$ unless $r-2 k=2$ or 0 . Hence (ii) is deduced without difficulty. In the other case, we find

$$
\sum_{p \leqq x}(2 \cos \theta(p))^{2 r}=\left(-\left(\begin{array}{c}
2 r \\
r-1
\end{array}\right)+\left(\begin{array}{c}
2 r \\
r
\end{array}\right)\right)(1+o(1)) \frac{x}{\log x}
$$

as $x \rightarrow \infty$. The term in brackets is easily seen to be $\frac{1}{r+1}\left(\begin{array}{c}2 r \\ r\end{array}\right)$, as desired.

We will need the following combinatorial identities.

\section{Lemma 3.}

$$
\begin{gathered}
\sum_{j=0}^{r}(-1)^{j}\left(\begin{array}{l}
r \\
j
\end{array}\right)\left(\begin{array}{c}
2 j \\
j
\end{array}\right) \frac{1}{j+1} 2^{-2 j}=2^{-2 r-1}\left(\begin{array}{c}
2 r+2 \\
r+1
\end{array}\right), \\
\sum_{j=0}^{r}(-1)^{j}\left(\begin{array}{l}
r \\
j
\end{array}\right)\left(\begin{array}{c}
2 j+2 \\
j+1
\end{array}\right) \frac{1}{j+2} 2^{-2 j}=2^{-2 r} \frac{1}{r+2}\left(\begin{array}{c}
2 r+2 \\
r+1
\end{array}\right) .
\end{gathered}
$$

Proof. (i) can be found in Riordan [12, p. 120], (ii) can be deduced easily from (i) in the following way. Denote the sum in (i) by $S(r)$. Considering $S(r+1)-S(r)$ and using the identity

$$
\left(\begin{array}{c}
r+1 \\
j
\end{array}\right)-\left(\begin{array}{l}
r \\
j
\end{array}\right)=\left(\begin{array}{c}
r \\
j-1
\end{array}\right)
$$

(ii) is deduced easily after a change of variable and some simplifications.

Proof of Theorem 4. (i) Consider the polynomial

$$
P_{m}(x)=\left(x^{2}-4\right)^{m}(x-\alpha)(x-\beta),
$$

where $\alpha, \beta$ will be suitably chosen later. By Lemmas 2 and 3 ,

$$
\frac{\log x}{x} \sum_{p \leqq x} P_{m}(2 \cos \theta(p)) \sim(-1)^{m}\left(\begin{array}{c}
2 m+2 \\
m+1
\end{array}\right)\left(\frac{\alpha \beta}{2}+\frac{1}{m+2}\right) .
$$

Examining the graph of $P_{m}(x)$ and choosing $\alpha, \beta$ so that

$$
\alpha \beta>-\frac{2}{m+2},
$$

if $m$ is even, and

$$
\alpha \beta<-\frac{2}{m+2},
$$

if $m$ is odd, we set $\alpha=-\delta$ to get the desired result.

(ii) Consider $Q_{m}(x)=x^{2 m}\left(x^{2}-\gamma\right)$ where $\gamma$ shall soon be chosen. By Lemma 2,

$$
\frac{\log x}{x} \sum_{p \leqq x} Q_{m}(2 \cos \theta(p)) \sim \frac{1}{m+2}\left(\begin{array}{c}
2 m+2 \\
m+1
\end{array}\right)-\gamma \frac{1}{m+1}\left(\begin{array}{c}
2 m \\
m
\end{array}\right) \text {. }
$$


Examining the graph of $Q_{m}(x)$, we note that if

$$
\gamma=\frac{m+1}{m+2}\left(\begin{array}{c}
2 m+2 \\
m+1
\end{array}\right)\left(\begin{array}{c}
2 m \\
m
\end{array}\right)^{-1}=\frac{2(2 m+1)}{m+2},
$$

we obtain (ii).

(iii) Since

$$
\sum_{p \leqq x}|2 \cos \theta(p)|^{2 m+1} \geqq \frac{1}{2} \sum_{p \leqq x}(2 \cos \theta(p))^{2 m+2}
$$

we have by Lemma 2 ,

$$
4 \sum_{\substack{p \leq x \\
a(p)>0}}(2 \cos \theta(p))^{2 m+1} \gtrsim \frac{1}{m+2}\left(\begin{array}{c}
2 m+2 \\
m+1
\end{array}\right) \frac{x}{\log x}
$$

as $x \rightarrow \infty$. Thus for a positive proportion of the primes,

$$
2 \cos \theta(p)>\left\{\frac{1}{4(m+2)}\left(\begin{array}{c}
2 m+2 \\
m+1
\end{array}\right)\right\}^{\frac{1}{2 m+1}}-\varepsilon .
$$

This completes the proof of the theorem.

Remark. We note that $\beta_{m} \rightarrow 2$, as $m \rightarrow \infty$. It is an interesting question to ask for the optimal value of $\beta_{m}$ under the hypothesis of the theorem. By considering various polynomials, one can show

$$
2 \cos \theta(p)>1.14-\varepsilon
$$

for a positive density of primes, with the present state of knowledge. V. Kumar Murty has informed me that if $L_{6}(s)$ has an analytic continuation up to $\operatorname{Re} s \geqq \frac{1}{2}$, then one can show

$$
2 \cos \theta(p)>1.33-\varepsilon
$$

for a positive density of the primes.

\section{Sign Changes of $a(p)$}

The classical method of analytic number theory gives a zero-free region of the type

$$
\sigma>1-A / \log (|t|+2), \quad A>0,
$$

for $L_{1}(s)$ and $L_{2}(s)$. This fact enables us to deduce:

Lemma 4. Let

$$
f(z)=\sum_{n=1}^{\infty} a(n) e^{2 \pi i n z}
$$


by a Hecke eigenform of weight $k$ for the full modular group. Then, there is a $v>0$, such that if $h=x^{\theta}, v<\theta<1$, we have

$$
\begin{gathered}
\sum_{x \leqq p \leqq x+h} a(p) p^{-(k-1) / 2}=o(h), \\
\sum_{x \leqq p \leqq x+h} a(p)^{2} p^{-(k-1)} \gg h .
\end{gathered}
$$

Proof. (ii) is contained in Moreno [7], and (i) is easily obtained from methods of that paper.

Let $g$ be another normalised Hecke eigenform of weight $k$ for the full modular group. Let $g$ have Fourier expansion

$$
g(z)=\sum_{n=1}^{\infty} b(n) e^{2 \pi i n z} .
$$

Then, Rankin showed that the series

$$
L(s, f, g)=\sum_{n=1}^{\infty} a(n) b(n) n^{-s-k+1}
$$

has an analytic continuation to $\operatorname{Re} s \geqq \frac{1}{2}$ and satisfies a functional equation.

For any eigenform with Fourier expansion

$$
h(z)=\sum_{n=1}^{\infty} c(n) e^{2 \pi i n z}
$$

define $L_{2}(s, h)$ as the $L_{2}(s)$ defined in Sect. 1. The next lemma concerns the nonvanishing of $L(s, f, g)$ on $\sigma=1$.

Lemma 5. For $f, g$ orthogonal, normalised Hecke eigenforms of weight $k$,

(i) $L(1+i t, f, g) \neq 0$,

(ii) $L(s, f, g) \neq 0$ for $\sigma>1-C / \log (|t|+2)$, for some $C>0$,

(iii) there is a $v$ such that if $h=x^{\theta}, v<\theta<1$, we have

$$
\sum_{x \leqq p \leqq x+h} a(p) b(p) p^{-(k-1)}=\underline{o}(h) .
$$

Proof. We shall prove (ii) from which (i) follows. (iii) is then easily deduced from the methods of Moreno [7].

Consider

$$
D(s)=\zeta^{2}(s) L_{2}(s, f) L(s+i t, f, g) L(s-i t, f, g) L_{2}(s, g) .
$$

Then, an easy verification shows that

$$
\log D(s)=\sum_{n, p} C(n, p) n^{-1} p^{-n s},
$$

where $C(n, p)=8(\cos n \theta(p))(\cos n \phi(p))(\cos n t \log p)+2 \cos 2 n \theta(p)+2 \cos 2 n \phi(p)+4$.

In view of the identity

$$
C(n, p)=4\left|\cos \theta(p)-p^{i t} \cos \phi(p)\right|^{2}
$$


we see that $\log D(s)$ is a Dirichlet series with non-negative coefficients. Therefore, for $\sigma>1$

$$
D(\sigma)=\zeta^{2}(\sigma) L_{2}(\sigma, f) L(\sigma+i t, f, g) L(\sigma-i t, f, g) L_{2}(\sigma, g) \geqq 1
$$

taking logarithmic derivatives and using the fact that $L_{2}(s, f), L(s, f, g), L_{2}(s, g)$ are regular in $\sigma \geqq \frac{1}{2}$, we find by the standard method that $L(s, f, g)$ is zero-free in $\sigma>1$ $-\frac{C_{1}}{\log (|t|+2)}$, for some $C_{1}>0$. This completes the proof.

We now prove Theorem 5 .

Proof of Theorem 5. For suitable complex numbers $e_{i}, i=1, \ldots, d$, where $d=\operatorname{dim} S_{k}$ (the space of cusp forms of weight $k$ for the full modular group), we can write

$$
a(p)=\sum_{i=1}^{d} e_{i} a_{i}(p)
$$

where

$$
f_{i}(z)=\sum_{n=1}^{\infty} a_{i}(n) e^{2 \pi i n z}
$$

is a normalised Hecke eigenform.

Suppose that neither $\operatorname{Re} a(p)$ nor $\operatorname{Im} a(p)$ changes sign in $x \leqq p \leqq x+h$, for $h=x^{\theta}$, where $\theta$ satisfies the conditions of Lemmas 4 and 5(iii). Put $a_{p}=a(p) p^{-(k-1) / 2}$. Then,

$$
\left|\sum_{x \leqq p \leqq x+h} \operatorname{Re} a_{p}\right|=\sum_{x \leqq p \leqq x+h}\left|\operatorname{Re} a_{p}\right|
$$

and

$$
\left|\sum_{x \leqq p \leqq x+h} \operatorname{Im} a_{p}\right|=\sum_{x \leqq p \leqq x+h}\left|\operatorname{Im} a_{p}\right|
$$

Therefore,

$$
\sum_{x \leqq p \leqq x+h}\left(\left|\operatorname{Re} a_{p}\right|+\left|\operatorname{Im} a_{p}\right|\right)=\underline{o}\left(x^{\theta}\right) .
$$

On the other hand, by the estimate of Deligne, the left hand side of (1) is

$$
\gg \sum_{x \leqq p \leqq x+h}\left|a_{p}\right|^{2}=\sum_{1 \leqq i, j \leqq d} e_{i} \bar{e}_{j} \sum_{x \leqq p \leqq x+h} a_{i}(p) a_{j}(p) p^{-(k-1)} \gg h \equiv x^{\theta},
$$

by Lemma 4(ii). This is a contradiction to (1). Therefore, at least one of $\operatorname{Re} a(p)$ or Im $a(p)$ changes sign in the interval $x \leqq p \leqq x+h$. This completes the proof of Theorem 5 .

\section{Main Theorems}

By Corollary 2 of Theorem 4, we have

$$
|a(p)|>(\sqrt{2}-\varepsilon) p^{\frac{k-1}{2}}
$$


for a positive proportion of the primes. So we must have

$$
a(p)>(\sqrt{2}-\varepsilon) p^{\frac{k-1}{2}}
$$

or

$$
a(p)<(-\sqrt{2}-\varepsilon) p^{\frac{k-1}{2}}
$$

for a positive proportion of the primes. Without loss, suppose that (1) is true.

Let

$$
n=\prod_{p \leqq x}^{*} p
$$

where the product is over those primes satisfying (1).

Then

$$
a(n)>n^{\frac{k-1}{2}}(\sqrt{2}-\varepsilon)^{\delta x / \log x}
$$

for some $\delta>0$. As $\log n \sim \delta x$ we get

$$
a(n)>n^{\frac{k-1}{2}} \exp \left(\frac{C \log n}{\log \log n}\right)
$$

for some $C>0$ and an infinity of $n$. If $q$ is a prime such that $a(q)<0$ [which exists either by Theorem 4(iii) or Theorem 5], the $n$ 's constructed above are coprime to $q$ and hence

$$
-a_{q n}=\left(-a_{q}\right) a_{n} \gg n^{\frac{k-1}{2}} \exp \left(\frac{C \log n}{\log \log n}\right),
$$

which completes the proof of Theorem 1.

Proof of Theorem 2. Let $f_{1}, \ldots, f_{r}$ be a basis of eigenforms. Then there are complex numbers $C_{1}, \ldots, C_{r}$ such that

$$
f(z)=\sum_{i=1}^{r} C_{i} f_{i}(z)
$$

Let

$$
f_{j}(z)=\sum_{n=1}^{\infty} a(n, j) e^{2 \pi i n z}
$$

The matrix $(a(j, i))$ for $j \leqq r, i \leqq r$ is a non-singular matrix ; for if not we can find $d_{1}, \ldots, d_{r}$ not all zero such that

$$
d_{1} f_{1}+\ldots+d_{r} f_{r}=f_{0}
$$

has a zero of order $\geqq r+1$ at $i \infty$. The dimension formula shows that $f_{0} \equiv 0$. But then, this contradicts the independence of the $f_{j}$ 's. Hence, we can find $m_{1}, \ldots, m_{r}$ so that

$$
\sum_{j=1}^{r} a(j, i) m_{j}= \begin{cases}1 & i=1 \\ 0 & i \neq 1\end{cases}
$$


Now consider

$$
g(z)=\sum_{i=1}^{r} m_{i} T_{i}(f)
$$

where $T_{i}$ is the $i^{\text {th }}$ Hecke operator. Then

$$
\begin{aligned}
g(z) & =\sum_{i=1}^{r} m_{i} \sum_{j=1}^{r} C_{j} a(i, j) f_{j}(z) \\
& =\sum_{j=1}^{r} C_{j} f_{j}(z) \sum_{i=1}^{r} a(i, j) m_{i}=C_{1} f_{1}(z) .
\end{aligned}
$$

On the other hand, the $n^{\text {th }}$ Fourier coefficient of $g(z)$ is

$$
=\sum_{i=1}^{r} m_{i} \sum_{d \mid(n, i)} a\left(n i / d^{2}\right) d^{k-1}
$$

by noting what the Hecke operators do to the Fourier coefficients. Now suppose, that for all $n$ sufficiently large,

$$
|a(n)|<\varepsilon n^{\frac{k-1}{2}} \exp \left(\frac{C \log n}{\log \log n}\right) .
$$

Then, if $d(i)$ denotes the number of divisors of $i$,

$$
\begin{aligned}
\left|C_{1} a(n, 1)\right| & \leqq \sum_{i=1}^{r}\left|m_{i}\right| i^{k-1} \varepsilon d(i) n^{\frac{k-1}{2}} \exp \left(\frac{C \log n}{\log \log n}\right) \\
& \ll \varepsilon n^{\frac{k-1}{2}} \exp \left(\frac{C \log n}{\log \log n}\right)
\end{aligned}
$$

which contradicts our previous theorem for suitable $C$ and $\varepsilon$.

Remark. Conjecture 1 is certainly true whenever $k=12,16,18,20,22,26$ (see Sect. 6). In case $k=24$, one can compute $m_{1}$ and $m_{2}$ above and find that $m_{1}>0$, $m_{2}>0$. This enables one to show Conjecture 1(ii) by the above method, in this case also.

We can now prove the corollary on Kloosterman sums.

Proof of Corollary. It is well-know that the Poincare series

$$
G_{t}(z)=\frac{1}{2} \sum_{\langle c, d)=1}(c z+d)^{-k} e^{2 \pi i t(a z+b) /(c z+d)}
$$

for $1 \leqq t \leqq r$, where $r$ is the dimension of the space of cusp forms of weight $k$, span this space. Moreover, the $n^{\text {th }}$ Fourier coefficient of $G_{t}(z)$ is

$$
(n t)^{\frac{k-1}{2}}\left\{\delta_{t n}+\pi \sum_{c=1}^{\infty} \frac{S(t, n, c)}{c} \cdot J_{k-1}\left(\frac{4 \pi \sqrt{n t}}{c}\right)\right\},
$$

where $S(t, n, c)$ is the Kloosterman sum defined previously and $J_{k}$ denotes the usual Bessel function. Set

$$
H(x)=\sum_{c \leqq x} S(n, m, c)
$$


Now suppose the corollary is false. Then, given $\varepsilon>0$,

$$
|G(x)| \leqq \exp \left(\frac{C \log x}{\log \log x}\right)
$$

for all $x$ sufficiently large and by partial summation,

$$
|H(x)| \leqq \varepsilon x \exp \left(\frac{C \log x}{\log \log x}\right) .
$$

It is easy to see that

$$
\begin{aligned}
& \sum_{c>\sqrt{n}} \frac{S(n, t, c)}{c} J_{k-1}\left(\frac{4 \pi \sqrt{n t}}{c}\right) \\
& =\sum_{c>\sqrt{n}} G(c)\left(J_{k-1}\left(\frac{4 \pi \sqrt{n t}}{c+1}\right)-J_{k-1}\left(\frac{4 \pi \sqrt{n t}}{c}\right)\right) \\
& \ll \sqrt{n} \sum_{c>\sqrt{n}} \frac{G(c)}{c^{2}} J_{k-1}^{\prime}\left(\xi_{c}\right),
\end{aligned}
$$

where $\xi_{c} \in\left(\frac{4 \pi \sqrt{n t}}{c+1}, \frac{4 \pi \sqrt{n t}}{c}\right)$. As $J_{k-1}^{\prime}(x) \ll x^{-1 / 2}$, we find that the above sum is

$$
\ll \varepsilon n^{-1 / 4} \int_{\sqrt{n}}^{\infty} \frac{e^{C \log t / \log \log t}}{t^{3 / 2}} d t
$$

An integration by parts reveals that it is

$$
\ll \varepsilon \exp \left(\frac{C \log n}{\log \log n}\right) \text {. }
$$

As there are no cusp forms of weight 10 , it follows from the above that

$$
\sum_{\varepsilon \leqq \sqrt{n}} \frac{S(n, t, c)}{c} J_{9}\left(\frac{4 \pi \sqrt{n t}}{c}\right) \ll \varepsilon \exp \left(\frac{C \log n}{\log \log n}\right) .
$$

Now

$$
\frac{1}{\sqrt{n}} \sum_{c \leqq \sqrt{n}} S(n, t, c) J_{10}\left(\frac{4 \pi \sqrt{n t}}{c}\right) \ll \sum_{c \leqq \sqrt{n}} \frac{H(c)}{c^{2}} J_{10}^{\prime}\left(\eta_{c}\right)
$$

for some $\eta_{c} \in\left(\frac{4 \pi \sqrt{n t}}{c+1}, \frac{4 \pi \sqrt{n t}}{c}\right)$. By our supposition, this sum is

$$
\ll n^{-1 / 4} \sum_{c \leqq \sqrt{n}} \frac{H(c)}{c^{3 / 2}} \ll \varepsilon \exp \left(\frac{C_{1} \log n}{\log \log n}\right)
$$

as $J_{k-1}^{\prime}(x) \ll x^{-1 / 2}$. But then, in view of the identity.

$$
\frac{20 J_{10}(x)}{x}=J_{9}(x)+J_{11}(x)
$$


we find,

$$
\sum_{c \leqq n} \frac{S(n, t, c)}{c} J_{11}\left(\frac{4 \pi \sqrt{n t}}{c}\right) \ll \varepsilon \exp \left(\frac{C_{1} \log n}{\log \log n}\right)
$$

for some constant $C_{1}>0$. This contradicts Theorem 2, which completes the proof of the corollary.

\section{Real Zeroes and Oscillations}

By standard techniques of analytic number theory, it is possible to show the following (see Ingham $[3,4]$ ).

Theorem 6. Let $f$ be a normalized eigenform of weight $k$, with Fourier expansion

$$
\sum_{n=1}^{\infty} a(n) e^{2 \pi i n z}
$$

Suppose $L_{1}(s)$ has no real zero in the interval $\frac{1}{2}<s \leqq 1$. Then

$$
\sum_{p \leqq x} a(p) p^{-(k-1) / 2}=\Omega_{ \pm}\left(x^{1 / 2} \log \log \log x / \log x\right) .
$$

Remark. Even if $L_{1}\left(\frac{1}{2}\right)=0$, it is of no consequence to the $\Omega$ result because the contribution from the zero is $O\left(x^{1 / 2} / \log x\right)$. It is easily seen that whenever $k \equiv 2$ $(\bmod 4), L_{1}\left(\frac{1}{2}\right)=0$, for instance.

One can show by means of the formula

$$
v_{\infty}+v_{i} / 2+v_{p} / 3+\sum_{P \neq i, p} v_{P}=k / 12,
$$

where $v_{p}$ denotes the order of the zero of $f$ at $P, i$ and $p$ denote $\sqrt{-1}$ and $e^{2 \pi i / 3}$. respectively, that $L_{1}(s)$ has no real zero in $\frac{1}{2}<s \leqq 1$ for $k<24$ and $k=26$. For $k=24$, it is possible to show the existence of at least one eigenfunction satisfying the hypothesis. We briefly indicate how this is done. Consider

$$
(2 \pi)^{-\left(s+\frac{1}{2} k-\frac{1}{2}\right)} \Gamma\left(s+\frac{1}{2} k-\frac{1}{2}\right) L_{1}(s)=\int_{1}^{\infty} f(i y) y^{(k-1) / 2}\left(y^{s}+i^{k} y^{1-s}\right) \frac{d y}{y} .
$$

It is then easy to show that the integral is non-negative for the specified values of $k$ using (3). For example, if $k=22$,

$$
v_{i} / 2+v_{p} / 3+\sum_{p \neq i, p} v_{p}=5 / 6,
$$

which clearly implies $f(i y) \geqq 0$. For $k=26, f$ is up to a constant multiple equal to $\Delta E_{4}^{2} E_{6}$ where $\Delta$ denotes Ramanujan,s cusp form, $E_{4}, E_{6}$ denote usual Eisenstein series. As we know the location of the zeroes of $E_{4}$ and $E_{6}$, the desired result follows in this case also. Moreover, (4) can be used to show that

$$
(2 \pi)^{-s} \Gamma(s) L_{1}\left(s-\frac{k-1}{2}\right)
$$

is a monotone increasing function for $s \geqq \frac{1}{2}$, just by expanding the integral about $s=\frac{1}{2}$. In fact, it seems reasonable to make the following conjecture. 
Conjecture 2. If $f$ is a normalized Hecke eigenform, then

$$
(2 \pi)^{-s} \Gamma(s) L_{1}\left(s-\frac{k-1}{2}\right)
$$

is a monotone increasing function of $s$ for $s \geqq \frac{1}{2}$.

Indeed, if $L_{1}(s)$ satisfies the analog of the Riemann hypothesis, the conjecture is true (see Stark and Zagier [16]).

\section{Concluding Remarks}

We discuss Conjecture 1(ii) as the first part of Conjecture 1 has been dealt with in the previous section.

The truth of the conjecture would follow if we postulate that the angles $\theta(p, i)$ for $i=1, \ldots, r$ corresponding to the basis of normalized Hecke eigenforms $f_{1}, \ldots, \hat{f}_{r}$, respectively, are independently distributed, with respect to the Sato-Tate measure. This can be cast, in the familiar way, in terms of $L$-series. For $\underline{m}=\left(m_{1}, \ldots, m_{r}\right)$, let

$$
f(p, \underline{m})=\sum_{a=1}^{r}\left(m_{a}-2 j_{a}\right) \theta(p, a)
$$

and consider

$$
L_{\underline{m}}(s)=\prod_{p} \prod_{j_{1}=0}^{m_{1}} \ldots \prod_{j_{r}=0}^{m_{r}}\left(1-\frac{e^{i f(p \cdot \underline{m})}}{p^{s}}\right)^{-1} .
$$

For $\underline{m}=(m, 0, \ldots, 0)$ we retrieve $L_{m}(s)$ as

$$
L_{\underline{m}}(s)=\left(L_{m}(s)\right)^{r}
$$

Clearly, $L_{m}(s)$ defines an analytic function for $\operatorname{Re} s>1$.

Conjecture 3. Each $L_{\underline{m}}(s)$ has an analytic continuation to the entire complex plane and $L_{\underline{m}}(1+i t) \neq 0$, for $m_{1}+\ldots+m_{r} \geqq 1$.

It may be remarked that if $m_{1}+\ldots+m_{r}=2$, then $L_{m}(s)$ is essentially the familiar convolution of $L$-series. Lemma 5 gives the non-vanishing of these $L$-series on $\sigma=1$.

Acknowledgements. I would like to thank Dr. V. Kumar Murty for several useful discussions especially in connection with Theorem 4. I would also like to thank Dr. S. Srinivasan and Professor $K$. Ramachandra for their useful comments, and the referee for several helpful suggestions.

\section{References}

1. Balasubramanian, R., Ram Murty, M.: An $\Omega$-theorem for Ramanujan's $\tau$-function. Invent. Math. 68, 241-252 (1982)

2. Hardy, G.H.: Ramanujan, New York: Chelsea 1940

3. Ingham, A.: The distribution of prime numbers. Cambridge: Cambridge University Press 1932

4. Ingham, A.: A note on the distribution of primes, Acta Arith. 1, 201-211 (1936)

5. Joris, $H$. : An $\Omega$-result for coefficients of cusp forms. Mathematika 22, 12 19 (1975) 
6. Linnik, U.V.: Additive problems and eigenvalues of the modular operators. Proc. of the Int. Congress, 1962, Sweden

7. Moreno, C.J.: The Hoheisel phenomenon for generalised Dirichlet series. Proc. Math. Soc. 40, 47-51 (1973)

8. Ram Murty, M. : Some $\Omega$-results for Ramanujan's $\tau$-function. Proceedings of the Mysore Number Theory Conference, 1981. In: Lecture Notes in Mathematics, Vol. 938. Berlin, Heidelberg, New York: Springer 1982

9. Murty, V.K.: On the Sato-Tate conjecture (to appear)

10. Ogg, A.P.: A remark on the Sato-Tate conjecture. Invent. Math. 9, 198-200 (1970)

11. Rankin, R.A.: An $\Omega$-result for the coefficients of cusp forms. Math. Ann. 203, 239-250 (1973)

12. Riordan, J.: Combinatorial identities. New York: Wiley 1968

13. Selberg, A.: On the estimation of Fourier coefficients of modular forms. Proc. Symp. Pure Math. 8, $1-15(1965)$

14. Serre, J.P.: Abelian 1-adic representations and Elliptic curves. New York: Benjamin 1968

15. Shahidi, F.: On certain $L$-functions. Am. J. Math. 103, 297-355 (1981)

16. Stark, H., Zagier, D. : A property of $L$-functions on the real line. J. Number Theory 12, 49-52 (1980)

Received May 21, 1982 